\title{
Autoregulatory and paracrine control of synaptic and behavioral plasticity by octopaminergic signaling
}

\author{
Alex C Koon, James Ashley, Romina Barria, Shamik DasGupta, Ruth Brain, Scott Waddell, \\ Mark J Alkema, and Vivian Budnik \\ Department of Neurobiology, University of Massachusetts Medical School, Worcester, \\ Massachusetts, USA
}

\begin{abstract}
Adrenergic signaling has important roles in synaptic plasticity and metaplasticity. However, the underlying mechanisms of these functions remain poorly understood. We investigated the role of octopamine, the invertebrate counterpart of adrenaline and noradrenaline, in synaptic and behavioral plasticity in Drosophila. We found that an increase in locomotor speed induced by food deprivation was accompanied by an activity- and octopamine-dependent extension of octopaminergic arbors and that the formation and maintenance of these arbors required electrical activity. Growth of octopaminergic arbors was controlled by a cAMP- and CREB-dependent positive-feedback mechanism that required Octp $\beta 2 R$ octopamine autoreceptors. Notably, this autoregulation was necessary for the locomotor response. In addition, octopamine neurons regulated the expansion of excitatory glutamatergic neuromuscular arbors through Octp $\beta 2 R s$ on glutamatergic motor neurons. Our results provide a mechanism for global regulation of excitatory synapses, presumably to maintain synaptic and behavioral plasticity in a dynamic range.
\end{abstract}

Synaptic plasticity is fundamental for an organism's ability to adapt to a changing environment. Adrenergic receptors and their ligands are key regulators of plasticity. Noradrenaline has been implicated in the retrieval of spatial and contextual memories ${ }^{1}$, and it enhances LTP by promoting the synaptic delivery of AMPA-type glutamate receptors $(\mathrm{GluR})^{2}$. Adrenergic signaling has also been implicated in the regulation of plasticity (also called metaplasticity) to reset a homeostatic circuit in response to acute perturbations, thus maintaining the circuit within a dynamic range ${ }^{3}$. However, the specific mechanisms by which adrenergic signals influence synaptic plasticity are poorly understood.

Octopamine, the invertebrate counterpart of adrenergic ligands, activates receptors that resemble adrenergic receptors ${ }^{4}$. Octopamine is important for appetitive reinforcement in honeybees $^{5}$ and flies 6,7 and modulates behaviors such as aggression ${ }^{8}$, egg-laying ${ }^{9}$, foodseeking ${ }^{10}$ and sleep ${ }^{11}$, as well as synaptic functions ${ }^{12}$.

(C2011 Nature America, Inc. All rights reserved.

Correspondence should be addressed to V.B. (vivian.budnik@umassmed.edu).

Note: Supplementary information is available on the Nature Neuroscience website.

AUTHOR CONTRIBUTIONS

A.C.K. designed and performed most experiments and contributed to manuscript writing; J.A. contributed to tool development, electrophysiology, experimental design and manuscript writing; R.B. performed RT-PCR and some immunocytochemistry; S.W., S.D. and R.B. generated, characterized and validated PACa function; M.J.A. helped with the design of the TBH antibody; and V.B. directed the project and wrote the manuscript in collaboration with A.C.K. and J.A.

COMPETING FINANCIAL INTERESTS

The authors declare no competing financial interests.

Reprints and permissions information is available online at http://www.nature.com/reprintsandpermissions/. 
The Drosophila larval neuromuscular junction (NMJ) is a powerful model system in which to investigate synaptic plasticity. Although glutamate is the primary excitatory neurotransmitter of the NMJ, larval NMJs are also innervated by octopaminergic motor neurons ${ }^{13}$. Larval NMJs show several forms of synaptic plasticity, such as continuous expansion during larval development to offset a massive increase in muscle size, as part of a homeostatic mechanism to maintain synaptic efficacy ${ }^{14}$. This process depends on signaling mechanisms such as the bone morphogenetic protein (BMP) $)^{15}$ and Wnt pathways ${ }^{16}$. Larval NMJs can also respond to changes in the environment such as food availability by rapid increases in synapse strength ${ }^{17,18}$. In addition, genetic and physiological manipulations that increase presynaptic activity promote synaptic expansion at $\mathrm{NMJs}^{19,20}$. To determine the relevance of octopaminergic innervation of body-wall muscles, we examined octopaminergic terminals during larval foraging behavior. Type II arbors responded to food deprivation by extending new endings. This effect depended on both activity levels and octopamine. Electrical activity at octopaminergic neurons was essential for initial and continued type II innervation of muscles. We uncovered a cAMP and CREB-dependent autoregulatory positive feedback mechanism that regulated the size of type II endings through the activation of Oct $\beta 2 \mathrm{R}$ autoreceptors. Type II innervation also regulated the plasticity of glutamatergic type I motor neurons through Oct $\beta 2 R$ s expressed in these neurons. Both the autocrine and paracrine mechanisms were required for the adaptive response to starvation.

\section{RESULTS}

\section{Locomotor increase associated with type II synaptic change}

Larval NMJs respond to acute changes in presynaptic activity by modifications in synaptic structure $^{20}$. However, the physiological conditions under which this mechanism is used by the intact organism are unknown. Larval foraging behavior is enhanced by food deprivation, which leads to long-lasting enhancement of evoked glutamate release from excitatory type I NMJs ${ }^{17}$. However, no gross changes in the structure of these endings have been observed ${ }^{17}$. Most body-wall muscles are co-innervated by at least one additional class of motor neuron, the octopaminergic type II motor neuron ${ }^{13}$ (Fig. 1a). Octopamine signaling has been implicated in appetitive behaviors and locomotion $6,10,21,22$. Therefore, to determine whether type II arbors changed structure during starvation, a physiological stimulus that increases locomotor activity, we labeled these arbors by expressing mCD8-GFP using a tyrosine decarboxylase-2 (Tdc2) promoter fused to Gat4 (Tdc2-Gal4; Fig. 1a). We imaged NMJs in intact early third-instar larvae live through the cuticle, deprived the larvae of food for $2 \mathrm{~h}$ and then imaged the same NMJs again (Fig. 1).

Food-deprived wild-type larvae showed a significant increase in locomotor speed compared to fed controls (Fig. 1c). Notably, type II endings in starved larvae showed dynamic filopodia-like extensions (synaptopods) that extended and retracted with a time course of minutes (Fig. 1b,d and Supplementary Movie 1). Although we also saw synaptopods before food deprivation ('natural synaptopods'; Fig. 1b), the number of synaptopods was significantly increased upon starvation (Fig. 1b,d). Thus, changes in locomotor activity were accompanied by structural changes at type II endings.

We next investigated whether type II endings were necessary for behavior. We eliminated octopaminergic neurons by expressing the cell-death protein head involution defective (Hid; Supplementary Fig. 1), which substantially reduced locomotor speed and the starvation response (Fig. 1e,f). A similar result has been observed in tyrosine beta-hydroxylase $\left(t b h^{\mathrm{nMI} 8}\right)$ and $t d c 2^{\mathrm{R} 054}$ mutants, which cannot synthesize octopamine ${ }^{9,23}$ (Fig. 1e,f). The defects in $t b h$ mutants were specific, as they were rescued by expressing a TBH transgene in octopaminergic neurons (Fig. 1e,f). Thus, the increase in locomotion elicited by food 
deprivation results in structural changes in octopaminergic endings, and octopamine innervation is necessary for this behavior.

We then investigated whether octopamine was sufficient to increase locomotor activity in the absence of starvation. We expressed channel rhodopsin-2 (ChR2) in octopaminergic neurons and stimulated the neurons with blue light before the locomotion assay. Lightstimulated animals showed a significant increase in locomotor speed and this effect was eliminated in $t b h$ mutants (Fig. 1g). Thus, octopamine neurons are necessary and sufficient to increase locomotion.

To determine whether octopaminergic innervation of body-wall muscles alone was sufficient to induce modifications in synaptic physiology, we applied exogenous octopamine to body-wall muscles devoid of central input. Bath application of $10 \mu \mathrm{M}$ octopamine elicited a 30\% increase in the amplitude of excitatory junctional potentials (EJPs) without any change in the amplitude of miniature EJPs (mEJPs; Fig. 1h,j). This was consistent with analysis of $t b h$ mutants, in which EJP amplitude was significantly decreased (Fig. 1i,j). Thus, changes in locomotor activity were accompanied by growth of synaptopods and probably by an increase in synaptic strength.

\section{Synaptopod extension preceded type II synapse formation}

We determined the physiological significance of synaptopods (Fig. 2) by examining their dynamics (Fig. 2a and Supplementary Movie 1). Many synaptopods formed varicosity-like structures at their tips, measuring $0.6 \pm 0.04 \mu \mathrm{m}$ in diameter (Fig. $2 \mathrm{~b}$ and Supplementary Movie 2), smaller than mature type II boutons $(1.57 \pm 0.05 \mu \mathrm{m})$. When new varicosities formed, synaptopod motility halted (Supplementary Movie 2), the varicosity enlarged and sometimes a new motile synaptopod emerged from that varicosity (secondary synaptopod; Fig. 2c and Supplementary Movie 3). Thus, synaptopod formation could be a mechanism for type II arbor extension. We investigated this possibility by examining the same type II NMJs from first- to third-instar larval stage. First-instar type II arbors had synaptopods (Fig. 2d, for example, red arrow; Supplementary Fig. 2a) and synaptopods containing a varicosity at their tips (Fig. 2d, for example, yellow arrowhead in inset; Supplementary Fig. 2a). These structures developed into a completely new or extended a type II branch (Fig. $2 \mathrm{~d}$ and Supplementary Fig. 2a). To show that newly formed varicosities corresponded to new boutons, we imaged larvae expressing both mCherry and synaptotagmin-1 (Syt1)-GFP as above. Syt1-GFP accumulated in the newly formed varicosities (Fig. 2e). Thus, synaptopod extension is a mechanism for expanding type II arbors, both during an acute increase in locomotor speed and during larval development.

We also labeled preparations with antibodies to different synaptic markers. FasciclinII (FasII) was present at $100 \%$ of synaptopods from the earliest stages (Fig. $2 \mathrm{f}$ and Supplementary Fig. 2b), and was maintained throughout subsequent stages of bouton maturation (Fig. 2l,m). By contrast, we found Syt1 in synaptopods only $40 \%$ of the time (Fig. 2g,1,m and Supplementary Fig. 2c) but it was always present at the onset of varicosity formation (Fig. 21,m and Supplementary Fig. 2d), suggesting that vesicles begin to traffic into synaptopods even before the formation of type II varicosities.

We determined the onset of octopamine synthesis using antibodies to TBH (Supplementary Fig. 3a-f) and found that TBH was never observed before the onset of new varicosity formation (Fig. 2h,1,m and Supplementary Fig. 2b-f), which suggests that the accumulation of another type of synaptic vesicle, marked by Syt1, preceded the accumulation of TBHcontaining vesicles. We identified active zones using antibodies to Bruchpilot (Brp; Elks/ Cast/Erc homolog $)^{24}$, which was observed in $27 \%$ of enlarged varicosities and after the appearance of $\mathrm{TBH}^{20,25,26}$ (Fig. 2i,1,m and Supplementary Fig. 2e). Finally, we observed the 
MAPI B-related protein Futsch only after a secondary varicosity was formed, but the immunoreactivity was punctate (Fig. 2j,1,m and Supplementary Fig. 2f). We could not determine when postsynaptic GluRs were first present, as the immunoreactivity was very low (Supplementary Fig. 4a). The above data further support the notion that synaptopod extension constitutes a mechanism for the formation of new type II synaptic boutons. Even in intact larvae, many of the synaptopods that formed after starvation developed varicosities (Fig. 2k). These observations also show that the formation of type II boutons follows a precise sequence of synaptic protein addition (Fig. 2l).

\section{Acute activity and octopamine initiate type II outgrowth}

The structural changes observed at type II boutons in intact larvae raised the possibility that octopaminergic neurons were activated during food deprivation or increased locomotion, leading to the expansion of type II arbors. We tested this hypothesis by increasing motor neuron activity either with high- $\mathrm{K}^{+}$-induced depolarization or by blue light stimulation of ChR2 expressed in octopaminergic neurons (Fig. 3). We subjected preparations to spaced stimulation, in which 5 cycles of stimulation with high- $\mathrm{K}^{+}$or blue light were separated by 15 -min rest periods ${ }^{20}$. We imaged identified type II endings before and after stimulation. Stimulated samples showed a significant increase in synaptopod number at type II endings after stimulation (Fig. 3a,c). As in intact larvae, unstimulated preparations also contained natural synaptopods, albeit at a lower frequency (Fig. 3a,b). Thus, similar to the starvation response, synaptopods at type II endings increased in frequency in response to spaced stimulation, and stimulation of octopamine neurons alone was sufficient to elicit this response. This was confirmed by genetically increasing activity at octopaminergic neurons by expressing a dominant-negative Shaker $\mathrm{K}^{+}$channel subunit $(\mathrm{ShDN})^{27}$ in type II motor neurons of an eag $\mathrm{K}^{+}$channel subunit mutant, which resulted in an increase in natural synaptopods (Fig. 3d).

Next, we sought to determine whether octopamine signaling could underlie the effects of activity. The elimination of octopamine in $t b h$-null mutants resulted in a significant decrease in natural synaptopods (Fig. 3d). By contrast, bath application of octopamine for $15 \mathrm{~min}$ to wild-type preparations resulted in a dose-dependent increase in the number of synaptopods, whereas tyramine application had no effect (Fig. 3b,e,f). Induction of synaptopods by octopamine required normal $(1.5 \mathrm{mM}) \mathrm{Ca}^{2+}$ levels, as decreasing $\mathrm{Ca}^{2+}$ to $0.1 \mathrm{mM}$ prevented this effect (Fig. 3e, sub-Ca ${ }^{2+}$ ).

We determined the relationship between activity and octopamine by stimulating the terminals with activity or octopamine at levels that did not reach the threshold for induction of synaptopods. If the effect of activity was to increase octopamine release, then presenting both subthreshold stimuli together should elicit significant synaptopod formation. The subthreshold stimuli consisted of three cycles of spaced depolarization and application of 10 $\mu M$ octopamine in $0.1 \mathrm{mM} \mathrm{Ca}^{2+}$, both of which were insufficient to induce synaptopods when presented alone (Fig. 3g). When applied together, however, they increased synaptopods to a level similar to that induced by five cycles of stimulation alone (Fig. 3g). Thus, exogenous octopamine can overcome the effect of insufficient activity for the induction of synaptopods and vice versa, consistent with the notion that synaptopod formation is the result of activity-dependent octopamine release.

Octopamine failed to induce synaptopods in $t b h$ mutants (Fig. 3e). Given that $t b h$ mutants have an accumulation of tyramine ${ }^{9}$ that might be developmentally deleterious, we also tested $t d c 2$ mutants, which lack tyramine accumulation. In $t d c 2$ mutants the response to octopamine was normal (Fig. 3e), suggesting that in $t b h$ mutants the accumulation of tyramine renders the NMJs insensitive to exogenous octopamine. 
We also examined synaptic growth by counting the number of type II boutons at the last stage of larval development. Increasing activity through expression of ShDN in octopaminergic neurons of eag mutants led to a significant increase in the number of type II boutons and terminal branches (Fig. 3h and Supplementary Fig. 5a). By contrast, in tbh mutants the number of type II boutons and branches was decreased (Fig. $3 \mathrm{~h}$ and Supplementary Fig. 4b). These phenolypes were specifically rescued by expressing a $t b h$ transgene in octopaminergic neurons (Fig. 3h and Supplementary Fig. 4b). The defect in $t b h$ mutants was not due to the accumulation of tyramine in these mutants, as a null mutant in $t d c 2$, which lacks both tyramine and octopamine, also showed a decrease in the number of type II boutons (Fig. 3h).

\section{Type II synaptogenesis and maintenance require activity}

We also used transgenic approaches to block activity at octopaminergic neurons (Fig. 4). We used constructs encoding ShiDN ${ }^{\text {ts }}$, which blocks vesicle recycling at restrictive temperatures ${ }^{28}$, EKO, a hyperpolarizing Shaker potassium channel ${ }^{29}$, and Kir2.1, which encodes an inward-rectifying $\mathrm{K}^{+}$channel that prevents membrane depolarization ${ }^{30}$. We tested the efficiency of the blockade by examining the ability of adult females to lay eggs, as octopamine function is required for egg laying ${ }^{9}$. Only expression of Kir 2.1 completely blocked egg laying. Strikingly, it also resulted in the complete elimination of type II innervation (Fig. 4a,b). This was not due to a pathfinding or cell death defect because type II motor neuron axons, labeled with mCD8-GFP, were always observed in the segmental nerves (Fig. 4c,d). In 69\% of the nerves examined, these axons stalled in the segmental nerve. However, in $31 \%$ of the cases axons traveled the entire distance from the CNS and stalled close to the NMJ without innervating the muscles (Fig. 4d). Thus, in the absence of activity, type II endings could not innervate body-wall muscles.

Although ShiDN ${ }^{\text {ts }}$ did not completely eliminate octopaminergic function, expressing this transgene and rearing the animals at the restrictive temperature of $29{ }^{\circ} \mathrm{C}$ were sufficient to elicit marked abnormalities in the innervation of muscles by type II endings. These included markedly reduced type II arbors (Supplementary Fig. 5b), lack of innervation of muscles by type II arbors (Supplementary Fig. 5d), thinning of type II neurites (Supplementary Fig. $5 \mathrm{f}, \mathrm{h}$ ) and lack of TBH in some type II boutons (Supplementary Fig. 5h).

To determine whether there was a critical period in which activity was required for type II innervation, and whether the lack of innervation was the result of activity-dependent synaptogenesis or degeneration, we determined when type II innervation was established during the larval period and the consequences of blocking activity at these stages. We first observed type II varicosities during the first-instar stage (Fig. 4e,g). Blocking activity eliminated type II boutons at any larval stage (Fig. 4f,h), suggesting that the absence of activity in type II motor neurons prevents synaptogenesis.

We also ubiquitously expressed a temperature-sensitive Gal80, which at $18{ }^{\circ} \mathrm{C}$ blocks Gal4mediated expression ${ }^{31}$, and simultaneously expressed Kir2.1 in octopaminergic neurons. Larvae were raised at $18{ }^{\circ} \mathrm{C}$, and then switched to $29^{\circ} \mathrm{C}$ at different stages to permit expression of Kir2.1. Suppressing the activity of octopaminergic neurons $24 \mathrm{~h}$ before the third-instar stage did not elicit any abnormality in type II bouton morphology (Fig. 4i). By contrast, blocking activity starting from the late second-instar stage resulted in breaks in type II arbors (Fig. 4j). This phenotype was most pronounced when activity was blocked from the late first-instar stage (Fig. 4k-m). Thus, activity in type II endings was required for synaptogenesis, whereas prolonged periods of inactivity after innervation led to the degeneration of type II endings. 


\section{Octopamine-induced type II growth requires CAMP and dCREB}

The finding that octopamine induced the growth of type II arbors suggested that an autoregulatory mechanism controlled the formation of type II endings. Octopamine could be acting on autoreceptors at type II endings, or octopamine might activate a retrograde signal that promotes the growth of type II boutons. Octopamine receptors are G-protein-coupled receptors, which can increase $\mathrm{Ca}^{2+}$ or $\mathrm{cAMP}^{4,32}$. Previous studies suggested that increasing cAMP levels by a mutation in the phosphodisterase Dunce (Dnc) enhanced synaptic growth at all boutons ${ }^{33}$. Therefore we examined whether manipulating the levels of cAMP in type II motor neurons could influence synaptopod formation in response to octopamine (Fig. 5). Mutations in $d n c$ significantly enhanced the number of naturally occurring synaptopods at type II endings (Fig. 5a,b,d). This phenotype was rescued by expressing Dnc exclusively in the octopaminergic neurons of $d n c$ mutants or by a genomic duplication (Dp) of $d n c$ (Fig. $5 d$ ). Thus, octopamine motor neurons contain a cAMP pathway that can promote synaptopod formation.

These observations were confirmed by using a mutation in rutabaga (rut), which encodes an adenylate cyclase, and thereby decreasing cAMP levels. Flies with the $r u t^{2080}$ mutation had significantly fewer natural synaptopods than wild-type flies and this phenotype was rescued by expressing Rut at octopaminergic neurons (Fig. 5d).

To determine whether cAMP was acutely sufficient for synaptopod induction we elevated cAMP using a photoactivatable adenylate cyclase $(\mathrm{PACa})^{34}$. We expressed PACa in octopaminergic neurons and stimulated the preparation with blue light using a spaced procedure. There was a significant increase in the number of synaptopods (Fig. 5c,e and Supplementary Movie 4), which showed that acute changes in cAMP were sufficient to induce synaptopods.

To determine whether cAMP was downstream of activity and octopamine in the induction of synaptopods, we applied octopamine to $d n c$ and rut mutants. As described above, $d n c^{\mathrm{M} 14}$ mutants have more naturally occurring synaptopods than the wild type, whereas rut ${ }^{2080}$ mutants have fewer. In $d n c$ mutants we expected that the increase in synaptopods by octopamine would be occluded, as natural synaptopods are already saturated in this mutant. In rut mutants, we expected that the decrease in adenylate cyclase activity would render NMJs unresponsive to octopamine. Consistent with these predictions, octopamine failed to increase the number of synaptopods in the two mutants (Fig. 5f) By contrast, increasing cAMP by activating PACa in a $t b h$ mutant background still induced synaptopods (Fig. 5e). Thus the cAMP pathway is probably downstream of octopamine in synaptopod induction.

In long-term plasticity, the cAMP pathway has been associated with the activation of CREB leading to the transcription of genes that are required for the formation of new synapses ${ }^{35}$. We sought to determine whether the growth of type II endings in response to octopamine release required dCREB function. A dCREB dominant-negative transgene ( $C R E B d n /$ $d C R E B 2-b$ ) has been shown to block CREB function ${ }^{36}$; we expressed this transgene in octopamine neurons and found that it suppressed the increase in octopamine-induced synaptopod formation (Fig. 5g). Similarly, the translational inhibitor cycloheximide and the transcriptional inhibitor actinomycin-D completely suppressed octopamine-dependent synaptopod formation (Fig. 5g). Thus, the autoregulatory mechanism that initiates the formation of new type II boutons activates a cAMP cascade that depends on CREBmediated transcription.

As perturbations in the cAMP pathway altered synaptopod formation, and mutations that prevented an increase in synaptopod formation in response to octopamine also caused behavioral defects, we predicted that $d n c$ and rut mutants would show similar defects. 
Although $d n c^{\mathrm{M} 14}$ mutants showed decreased locomotor speed and rut mutants had normal locomotor speed (Fig. 5h), the response to starvation was blocked in both mutants, similar to tbh mutants (Fig. 5i). The behavioral defect in rut mutants was completely rescued by expression of a Rut transgene in octopaminergic neurons (Fig. 5i). Thus, normal cAMP levels in octopaminergic neurons are required for the response to starvation and, at least in the case of Rut, the starvation response can be separated from a defect in basal locomotion.

\section{Oct $\beta 2 R$ autoreceptors mediate the autoregulatory mechanism}

Presynaptic octopamine autoreceptors are plausible candidates for mediating autoregulation of synaptic structure. Four octopamine receptors have been identified in the Drosophila genome-OAMB, Oct $\beta 1 \mathrm{R} / \mathrm{OA} 2, \operatorname{Oct} \beta 2 \mathrm{R}$ and Oct $\beta 3 \mathrm{R}$. OAMB receptors are homologous to mammalian $\alpha$-adrenergic receptors and can increase levels of $\mathrm{Ca}^{2+}$ or cAMP ${ }^{4,32}$. Oct $\beta 1 \mathrm{R}$, Oct $\beta 2 R$ and Oct $\beta 3 R$ receptors have been less studied, but share similarities with mammalian $\beta$-adrenergic receptors and are thought to increase cAMP. We interfered with the function of OAMB and Oct $\beta 2 R$. In the $o a m b^{584}$ genetic null allele ${ }^{37}$, the number of natural synaptopods, the induction of synaptopods by octopamine and the number of type II boutons were normal (Fig. 6a-c).

To block the function of Oct $\beta 2 R$ we used a hypomorphic allele ${ }^{11}$ as well as RNA interference (UAS-Oct $\beta 2 R-R N A i)$. We also verified the expression of Oct $\beta 2 R$ in the nervous system and body-wall muscles (either pre- or postsynaptically) and the effectiveness of the RNAi transgene by RT-PCR (Supplementary Fig. 6a,b). In $o c t \beta 2 R$ mutants the number of natural synaptopods was significantly decreased (Fig. 6a), which indicates that Oct $\beta 2 R$ is the likely mediator of the cAMP-dependent autoregulatory mechanism that controls the growth of type II endings. Accordingly, oct $\beta 2 R$ mutants did not show increased synaptopods in response to octopamine (Fig. 6c). This effect was cell autonomous, as expressing Oct $\beta 2 \mathrm{R}-\mathrm{RNAi}$ in octopaminergic neurons alone was sufficient to decrease the number of natural synaptopods (Fig. 6a) and to suppress the ability of octopamine to induce an increase in synaptopods (Fig. 6c). In addition, either the $o c t \beta 2 R$ mutation or expression of Oct $\beta 2 R-R N A i$ in octopamine neurons resulted in a significant decrease in the number of type II boutons (Fig. 6b). We used C380-Gal4 to express Oct $\beta 2 R-R N A i$ in both type I and type II motor neurons, which also caused a significant reduction in the number of type II boutons (Fig. 6b). By contrast, the expression of Oct $32 \mathrm{R}-\mathrm{RNAi}$ in muscles using C57-Gal4 had no effect. These observations identify Oct $\beta 2 R$ receptors as the likely autoreceptor that regulates the growth of type II boutons.

The $o c t \beta 2 R$ mutant showed a reduction in evoked RJP amplitude similar to that seen in $t b h$ mutants (FJP amplitude, $18.3 \pm 1 \mathrm{mV}$ in wild type versus $12.1 \pm 1 \mathrm{mV}$ in $o c t \beta 2 R$ mutants; $n$ $=7, P<0.001$ ), which suggests that the removal of either octopamine or its receptors decreases synaptic strength. As expected, bath application of octopamine to oct $\beta 2 R$ mutants did not change EJP amplitude in mutants as it did in the wild type (ratio EJP amplitude is 0.95 upon $10 \mu \mathrm{M}$ octopamine application versus 1.33 in wild type; $n=5, P<0.005$ ). Thus, Oct $\beta 2 \mathrm{R}$ receptors are probably responsible for the octopamine-induced changes in synaptic strength.

We predicted that removing the receptor would also eliminate the starvation response. Flies with mutations in $o c t \beta 2 R$ failed to respond to starvation by increasing locomotor speed (Fig. $6 \mathrm{~d})$. This detect was also observed when Oct $\beta 2 \mathrm{R}$ was downregulated either in octopamine neurons or in both type I and type II motor neurons, but not in muscles (Fig. 6d). 


\section{Type II endings regulate type I synaptic bouton outgrowth}

Excitatory transmission at the larval NMJ is mediated by the release of glutamate from type 1 NMJs. Like type II arbors, type I arbors expand continuously throughout larval development, in strong correlation to muscle size ${ }^{38}$. We considered the possibility that type II innervation might regulate this form of plasticity at type I boutons. To test this possibility, we first eliminated type II boutons by expressing Hid in octopaminergic neurons. The absence of type II innervation led to a substantial reduction in the number of type I boutons (Fig. 7a and Supplementary Fig. 6c). We obtained similar results when we expressed Kir2.1 or in $t b h$ mutants (Fig. 7a). The reduction in the number of type I boutons in $t b h$ mutants was restored by expressing a $t b h$ transgene in octopamine neurons (Fig. 7a). These results suggest that type II innervation regulates the plasticity of type I endings and therefore that it is involved in a form of metaplasticity.

To further characterize the influence of type II endings on type I arbors, we examined oct $\beta 2 R$ mutants, and found a reduction in the number of type I boutons (Fig. 7b). A potential mechanism by which type II arbors might regulate the growth of type I endings is through the presence of octopamine receptors at type I boutons. Therefore, we used the C380-Gal4driver to downregulate Oct $\beta 2 \mathrm{R}$ in both type I and type II motor neurons or the BG439-Gal4 driver to express Gal4 in type I motor neurons but not in type II motor neurons (Supplementary Fig. 7). Both manipulations resulted in a significant decrease in the number of type I boutons (Fig. 7b). By contrast, the downregulation of Oct $\beta 2 \mathrm{R}$ in octopamine neurons alone did not result in a significant decrease in the number of type I boutons. These results suggest that Oct $\beta 2 \mathrm{R}$ is required in type I motor neurons for normal expansion.

\section{DISCUSSION}

Adrenergic signaling is involved in the regulation of synaptic plasticity ${ }^{39,40}$. However, the precise mechanisms of this regulation are poorly understood. We show that octopamine regulates behavioral and synaptic plasticity through an autoregulatory mechanism that promotes the growth of type II innervation and in turn the expansion of excitatory glutamatergic arbors. This process seems to be associated with physiological stimuli that lead to increased locomotion. We propose that food deprivation elicits the release of octopamine by type II terminals. Octopamine binds to Oct $\beta 2 R$ receptors and thereby increases cAMP, which activates CREB-dependent regulation of transcription and leads to new type II synaptic growth (Supplementary Fig. 8a). This autoregulatory mechanism might control the amount of octopamine released by type II arbors. In turn, octopamine release stimulates the growth of type I arbors through Oct $\beta 2 \mathrm{R}$ at type I motor neurons. This mechanism would regulate, in a global fashion, excitatory transmission at the NMJ (Supplementary Fig. 8a).

Increases in larval locomotion, type II motor neuron activity or exogenous octopamine resulted in the extension of synaptopods. With the demonstration that synaptopod extension constitutes a mechanism for the formation of type II boutons, these results suggest that the above events control the growth of octopaminergic endings in an acute manner. Analysis of mutations in octopamine receptors and components of the cAMP cascade revealed an autoregulatory mechanism that controls this growth. First, expression of Oct $\beta 2 \mathrm{R}$ in type II motor neurons was required for type II synaptic growth. Second, altering cAMP levels by mutations in $d n c$ or rut modified this response in a manner consistent with positive regulation by cAMP. This regulation was cell autonomous in octopaminergic motor neurons, as the defects in synaptopod formation and type II synaptic growth were also elicited or rescued by transgene expression in octopaminergic motor neurons alone, in a chronic or acute manner. The presence of auto-octopamine receptors had been suggested in locusts ${ }^{41}$, although the identity of the proposed autoreceptor was not known. However, it was 
proposed that the locust octopamine autoreceptors served to inhibit octopamine release. By contrast, our experiments are consistent with a positive feedback mechanism that enhances synaptic growth. Autoregulatory mechanisms that control the amount of neuromodulator release have been previously demonstrated for neuromodulators such as dopamine ${ }^{42}$.

As in other forms of synaptic plasticity, including late LTP and long-term memory ${ }^{35}$, the autoregulatory mechanism required the function of CREB and new protein synthesis. This finding underscores the universality of mechanisms by which the nervous system modifies the efficacy of connections in a long-lasting manner. Octopamine receptor activation leading to CREB signaling has also been demonstrated in Caenorhabditis elegans ${ }^{10}$.

Our studies showed that this pathway regulated the structure of octopaminergic arbors in an autoregulatory fashion, and that this influenced the growth of type I excitatory arbors. The presence of a positive feedback that controls the growth of modulatory inputs in an acute manner provides a mechanism by which animal experience can modify circuitry and thus by which animals can adapt to a changing environment.

Activity was absolutely required for innervation of body-wall muscles by type II arbors, as reduced activity perturbed type II synaptogenesis. This is in contrast to the widely held view that although activity is important for the refinement of connections, it is not required for initial synaptogenesis ${ }^{43}$. Part of this view arises from the examination of arbors that mediate classical neurotransmission ${ }^{43}$. By contrast, the dependence of modulatory terminal growth on activity has been less studied. Our studies provide compelling evidence that octopamine has an influence on bouton outgrowth in octopaminergic type II and type I motor neurons. Studies of type I bouton outgrowth have identified local factors that influence the development of pre- or postsynaptic compartments, including Wnts and BMPs ${ }^{15,16}$. We suggest that octopamine release by type II arbors might mediate a more global regulation of outgrowth.

At the Drosophila larval NMJ, glutamatergic type Ib motor neurons innervate each muscle in an approximately 1:1 manner ${ }^{44}$ (Supplementary Fig. 8b, type Ib). In addition, two glutamatergic type Is motor neurons innervate the entire ventral or dorsal muscle field within each hemisegment ${ }^{44,43}$ (Supplementary Fig. 8b, type Is). By contrast, the three octopaminergic neurons per segment innervate most of the body-wall muscles in a bilateral fashion $^{13}$ (Supplementary Fig. 8b, type II). The layout of this innervation suggests that type II synapses might establish global regulation of the plasticity of type I arbors. This might serve as a mechanism for setting excitability levels in the entire body wall, and thereby keep synaptic function in a dynamic range. Similarly, studies in mammalian systems have shown that adrenergic signaling can affect plasticity at glutamatergic synapses, either through changes in ionotropic GluR localization ${ }^{2}$ or through regulation of metabotropic GluR, which affects the ability of a synapse to become potentiated depending on its history ${ }^{3}$. Octopamine might regulate the ability of type I NMJs to trigger muscle contraction by long-term regulation of type I synaptic growth.

Two previous studies at the Drosophila larval NMJ have shown that octopamine enhances synaptic transmission 46,47 . However, another study reported that octopamine might inhibit glutamatergic transmission in first-inslar larvae ${ }^{48}$. Our studies suggest that blocking activity or interfering with octopamine signaling in type II neurons leads to a decrease in type I synaptic outgrowth, consistent with the idea that octopamine release is a positive regulator of type I transmission. We suggest that in the short term, octopamine enhances synaptic strength, as observed in our electrophysiology experiments, leading to the observed increase in crawling behavior after starvation. This would be consistent with studies showing that 
increases in locomotor speed induced by food deprivation led to an enhancement of synaptic efficacy $^{17}$.

Octopamine is a potent modulator of invertebrate behavior and is secreted during starvation in invertebrates ${ }^{10,22}$. Nevertheless, its function at the synaptic level is poorly understood. Our study shows that octopamine can influence synapses at the structural level through the activation of $\operatorname{Oct} \beta 2 \mathrm{R}$ autoreceptors in octopamine neurons and through the presence of these receptors in type I motor neurons.

An important question is whether octopamine is simply involved in locomotion and the lack of starvation response in mutants that cannot synthesize octopamine is an indirect effect of defective locomotion. It is not possible to answer this question in tbh mutants, as basal locomotion was reduced in these mutants. However, our experiments revealed conditions in which changes in basal activity could be genetically separated from changes in the starvation response. One such case is rut mutants, which have normal locomotion but lack the starvation response. This effect seemed to be due to the function of Rut in octopamine neurons, as the defective starvation response was completely rescued by expressing a Rut transgene in octopamine neurons. A second, albeit less clear observation regards oct $\beta 2 R$ mutants. Although baseline locomotion was much less altered in these mutants than in $t b h$ mutants, these animals still could not mount a starvation response (Fig. 6d,e). Thus, it is likely that octopamine neurons are involved not only in locomotion, but also in the response to starvation.

Octopamine is also required for appetitive memory in adult fruit flies ${ }^{7}$. Notably, the appetitive memory procedure requires starvation before the assay, and $t b h$ mutants cannot learn in this procedure. Octopamine has been proposed to mediate the reinforcing effects of sugar in appetitive memory formation ${ }^{5,7}$. Our studies raise the possibility that this mechanism might involve structural changes at synaptic sites.

Although our studies focused on structural changes at type II NMJs, many of our manipulations affected all octopamine neurons, as Tdc2-Gal4 drives Gal4 in all octopaminergic neurons. Thus, our studies cannot rule out an influence from other octopaminergic neurons, besides motor neurons, in the changes observed and in the behavior. However, the finding that the manipulations resulted in specific changes in type II NMJ terminals and that octopamine modulates synaptic strength at the NMJ argues that at least some of the effects are likely to be due to the peripheral octopamine innervation.

In summary, our studies reveal important mechanisms by which activity regulates the ability of motor neurons to scale the release of regulatory signals, which is important for the adaptation of the organism to the environment. In addition, they show a mechanism by which excitatory synapses are regulated in a global manner, presumably to maintain synaptic plasticity in a dynamic range.

\section{ONLINE METHODS}

\section{Fly strains}

Flies were reared in standard Drosophila medium at $25^{\circ} \mathrm{C}$ except where indicated. Animals used in RNAi experiments were reared at $29^{\circ} \mathrm{C}$ to increase knockdown efficiency. The following stocks were used: the wild-type strain Canton-S (CS), Tdc2-Gal4 (Bloomington Stock Center), UAS-Hid ${ }^{49}, t b h^{\mathrm{nM} 18}$ (ref. 9), $t d c 2^{\text {RG54 }}$ (ref. 23), UAS-Kir2.1 (ref. 30), tubPGal80 ${ }^{\text {ts }}$ (Bloomington), UAS-ShDN ${ }^{27}$, eag ${ }^{1}$ (Bloomington), UAS-mCD8-GFP (Bloomington), $y d n c^{\mathrm{M} 14} c v f\left(\right.$ Bloomington)y $y w d n c^{\mathrm{ML}} f^{6 \mathrm{a}}$ (Bloomington), UAS-Dnc (remobilized to the second chromosome) ${ }^{50}$, rut $^{2080}$ (Bloomington), UAS-Rut 
(Bloomington), UAS-dCRF.B2-b (Bloomington), $o a m b^{584} \mathrm{Pbac}\{\mathrm{WH}\} \mathrm{Oct} \beta 2 \mathrm{R}[\mathrm{f05679]}$

(Bloomington), Dp(1;2)51b (duplication of $d n c$; Bloomington), UAS-Syt1-GFP

(Bloomington), C380 (Budnik), BG439 (V. Budnik, unpublished), UAS-Dicer-2

(Bloomington), UAS-Octß2R-RNAi (8486 and 104524; Vienna Drosophila RNAi Center)

and UAS-PACa (see below).

\section{Generation of PACa flies}

Euglena gracilis PACa cDNA was provided by M. Watanabe. A 3,104-bp EagI fragment encompassing the full-length PACa cDNA with $5^{\prime}$ leader sequence was ligated into the EagI site of the Drosophila transformation vector pUAST. The insert was verified to be in the appropriate orientation by PCR and end sequencing, and transformed into flies by germline transformation.

\section{Generation of TBH antibodies}

TBH antibodies were raised against amino acids $277-670$ of TBH, which is the following peptide sequence: QETTYWCHVQR LEGNLRRRHHIVQFEPLIRTPGIVHHMEVFHCEAGEHEEIPLYNGDCEQLPPRAKICS KVMVLWAMGAGTFTYPPEAGLPIGGPGFNPYVRLEVHFNNPEKQSGLVDNSGFRIK MSKTLRQYDAAVMELGLEYTDKMAIPPGQTAFPLSGYCVADCTRAAIPATGIIIFGS QLHTHLRGVRVLTRHFRGEQELREVNRDDYYSNHFQRMRTLHYKPRVLPGDALVT TCYYNTKDDKTAALGGFSISDEMCVNYIHYYPATKLEVCKSSVSEETLENYFIYMK RTEHQHGVHLNGARSSNYRSIEWTQPRIDQLYTMYMQEPLSMQCNRSDGTRFEGR SSWEGVAATPVQIRIPIHRKLCPNYNPLWLKPLEKGDCDLLGECIY The specificity of the antibody was demonstrated by the lack of immunorcactivity in null $t b h^{\mathrm{nM} 18}$ mutants (Supplementary Fig. 1c,f).

\section{Immunocytochemistry}

Larval body-wall muscles were dissected and fixed for $15 \mathrm{~min}$ in $4 \%$ paraformaldehyde. For TBH immunocytochemistry samples were fixed in Bouin's fixative. Antibodies and their concentrations were: anti-TBH 1:400, anti-mCD8a 1:100 (Invitrogen), anti-HRP-Texas Red 1:200 (Jackson ImmunoResearch), anti-FasII 1:2 (Developmental Studies Hybridoma Bank, DSHB), anti-Syt1 1:100 (gift from T. Littleton), anti-Bruchpilol 1:100 (nc82; DSHB), antiFutsch 1:50 (22C10, DSHB), anti-GluRIIA 1:10 (8B4D2; DSHB). Secondary antibodies conjugated to FITC, Texas Red (Jackson) or Alexa 647 (Invitrogen) were used at a concentration of 1:200. Imaging of fixed preparations was as described ${ }^{20}$.

\section{Animal rearing conditions for synaptopod analysis}

All animals used in syn-aptopod analysis carried a copy of Tdc2-Gal4 and a copy of UASCD8:GFP. Egg collection was done in standard 25-mm diameter cornmeal/agar/molasses food vials at $25{ }^{\circ} \mathrm{C}$ with $-60 \%$ humidity, and larvae were kept at low density. Wandering late third-instar larvae were used for experiments.

\section{Stimulation procedures and live imaging of dissected preparations}

Synaptopods were imaged from live preparations as described ${ }^{20}$. Both the spaced high $\mathrm{K}^{+}$ depolarization procedure and the ChR2 stimulation procedure were as described ${ }^{20}$. Briefly, the high $\mathrm{K}^{+}$procedure consisted of 5 incubation cycles with $90 \mathrm{mM} \mathrm{K}^{+}$-containing saline, each lasting $2 \mathrm{~min}$ and separated by 15 -min resting intervals. For the ChR2 stimulation procedure, animals were placed inside a drop of HL3 saline $(\sim 300 \mu \mathrm{l})$ containing $1.5 \mathrm{mM}$ $\mathrm{Ca}^{2+}$. The procedure consisted of five cycles of blue light stimulation from four 491-nm 1W LEDs placed 1.2 meters away from the animals. Each cycle consisted of a repeating 5min procedure of $2 \mathrm{~s}$ on and $3 \mathrm{~s}$ off, separated by 15 -min resting intervals. Saline was 
exchanged once during each resting interval. Animals were imaged 15 min after stimulation or subjected to the crawling assay. For octopamine stimulation, larvae were dissected in HL3 saline containing $0.1 \mathrm{mM} \mathrm{Ca}^{2+}$ and preparations gently glued onto a custom-made glass imaging chamber using surgical glue. Then, identified NMJs were imaged on an Improvision spinning disc confocal microscope (PerkinElmer) with a C9100-13 Hamamatsu cooled EM-CCD camera and using a 40× $1.2 \mathrm{NA}$ objective, with a $2.4 \times$ optical zoom. After imaging for less than $30 \mathrm{~min}$, animals were partially unglued to allow muscles to contract freely, and $10 \mu \mathrm{M}$ octopamine in HL3 containing $1.5 \mathrm{mM} \mathrm{Ca}^{2+}$ was then applied for $15 \mathrm{~min}$ followed by $5 \times 15$-min washes with $0.1 \mathrm{mM} \mathrm{Ca}^{2+} \mathrm{HL} 3$ saline before imaging again. In some experiments actinomycin D (Sigma) $5 \mu \mathrm{M}$ in HL3, and cycloheximide (Sigma) 100 $\mu \mathrm{M}$ in IIL3 were applied throughout octopamine incubation and washes. The final concentration of DMSO in these solutions was approximately $0.03 \%$. For PACa experiments, NMJs were imaged as above, then stimulated with a broad spectrum blue light dental gun placed $\sim 2 \mathrm{~cm}$ away. Stimulation consisted of $5 \times 5$-s light exposures, each separated by 2 min rest.

\section{Live imaging of intact (undissected) larvae}

For live-imaging through the cuticle of intact larvae, animals were anesthetized using Sevoflurane (Baxter) and identified NMJs (muscle 9, A4) were imaged for not longer than $30 \mathrm{~min}$. Larvae were allowed to recover for $1.5 \mathrm{~h}$ on food plates and then used for food deprivation experiments (see below) before imaging. Larvae that did not recover in $1.5 \mathrm{~h}$ after the first imaging session or after starvation were discarded. For non-starved controls, larvae were placed in food between imaging sessions.

\section{Crawling assay}

Synchronized larvae were grown at $25^{\circ} \mathrm{C}$ in 28.5 - $\mathrm{mm}$ diameter standard food vials at low density until mid third-instar larval stage. After washing with water, individual larvae were loaded onto a $24 \times 24-\mathrm{cm} 3 \%$ agar plates and allowed a pre-run of $25 \mathrm{~s}$ on the agar before recordings were made. Larval tracks were then recorded manually for 1 min on transparency paper over the plate lid, and the distances crawled were measured using ImageJ. All behavioral experiments were carried out in a $25{ }^{\circ} \mathrm{C}, 60 \%$ humidity behavioral room, $n$ represents one animal. Percentage increase in locomotor speed of individual animals was calculated by: (locomotor speed of individual animals after starvation minus mean locomotor speed before starvation) $\times 100$ divided by the mean locomotor speed before starvation. Mean percentage and s.e.m. were calculated.

\section{Starvation assay}

Larvae were maintained in food or food-free moisturized 35-mm Petri dishes for $2 \mathrm{~h}$ and then either assayed for synaptopod formation or subjected to the crawling assay.

\section{Quantification of boutons and synaptopod number}

The number of type I boutons was obtained at muscles 6 and 7 of abdominal segment A3, and the number of type II boutons was measured at muscle 12 in A3. For muscle area measurements the muscle length and width were measured using an ocular scale bar. Measurements of synaptopod number were from muscle 12 (A4) in dissected preparations and those from intact larvae were from muscle 9 (A4). Numbers of synaptopods in the histograms represent the total number of synaptopods per $100 \mu \mathrm{m}$ of each arbor. Synaptopods were defined as such if they measured $0.5 \mu \mathrm{m}$ or more. $n$ represents number of NMJs. At most two NMJs (segment A3) were quantified in each animal. 


\section{Statistical analysis}

For comparisons between more than two sample groups an analysis of variance (ANOVA) with Tukey post-hoc test was performed. For pair-wise comparisons a Student t-test was used. Numbers in histograms represent mean \pm s.e.m. Unless otherwise noted, sample number $(n)$ represents one synaptic arbor for anatomical measurements, or one animal for behavioral analyses.

\section{Genotype abbreviations}

Type II driver control, Tdc2/+; Type I+II driver control, C380-Ga14/+; muscle driver control, C57-Gal4/+; [transgene]-type II, Tdc2-Gal4>[transgene];[transgene]-type I+II, C380-Gal4>[transgene];[transgene]-muscle, C57-Gal4>[transgene] unless otherwise indicated; Dcr, UAS-Dicer-2.

\section{Electrophysiology}

Membrane potential recordings were performed on dissected third-instar larvae as described ${ }^{20}$. Briefly, larvae were dissected in a custom magnetic chamber in $0.3 \mathrm{mM}$ $\mathrm{Ca}^{2+} \mathrm{HL} 3$ saline, and the A3 segmental nerves carefully cut close to the ventral ganglion. The chamber was then moved to the recording setup where it was perfused with $0.5 \mathrm{mM}$ $\mathrm{Ca}^{2+}$ HL3. Muscle 6 in segment A3 was impaled with a 15-20-M $\Omega$ electrode, and voltage recordings were collected with an Axoclamp2A amplifier (Molecular Devices), using Heka Pulse software (Heka). Only samples with resting membrane potentials between -60 and $-63 \mathrm{mV}$ were considered. For EJP recordings the segmental nerve was stimulated with a suction electrode at $0.3 \mathrm{~Hz}$, with a stimulus of $0.3 \mathrm{~ms}$ and sufficient voltage to evoke responses from both type I boutons. Four minutes of both $\mathrm{mEJP}$ and EJP data were recorded for each sample. Data was analyzed using Minianalysis software (Synaptosoft), and statistical analysis was done with Origin software (OriginLab). Bath application of octopamine was performed by changing the perfusion solution from $0.5 \mathrm{mM} \mathrm{Ca}^{2+} \mathrm{HL} 3$ to $0.5 \mathrm{mM} \mathrm{Ca}^{2+} \mathrm{HL} 3$ containing $10 \mu \mathrm{M}$ octopamine. Samples were allowed to equilibrate for 2 $\min$ in the new saline, and then evoked and spontaneous events were recorded again. Statistical analysis was done as above.

\section{RT-PCR}

Total mRNA was extracted from dissected larval body wall muscles or larval brains using a combination of Trizol (Invitrogen) and the RNeasy kit (Qiagen). Synthesis of cDNA for $+\mathrm{RT}$ reactions was performed using the Superscript III kit (Invitrogen), where -RT reactions lacked reverse transcriptase. The $+\mathrm{RT}$ and $-\mathrm{RT}$ reactions were then diluted and used for PCR using the forward primer CATGCTGATGCACCGACCATC and the reverse primer CACTCCTCGCAGGTCATGGAG. These primers were specifically designed to recognize all known splice variants of $o c t \beta 2 R$, and across two exon-intron junctions to avoid false signals from any contaminating genomic DNA. For semi-quantitative RT-PCR, we determined the linear range of Oct $\beta 2 R$ amplification for the amount of starting cDNA (50 ng) and number of PCR cycles for wild type, and then amplified cDNA made from RNA isolated from wild type and C380>UAS-Oct $\beta 2$ R-RNAi.

\section{Quantification of branch-points}

Type II arbors were examined in an epifluorescence microscope and each branch bifurcation was counted as a single branch point. For these quantifications $n$ is the number of total type II arbors (segment A3) quantified for each genotype. 


\section{Supplementary Material}

Refer to Web version on PubMed Central for supplementary material.

\section{Acknowledgments}

We thank M. Yoshihara, S. Speese, Y. Fuentes-Medel and C. Korkut for comments on the manuscript, C. Brewer for assistance with data analysis and the UMass Amherst antibody facility for production of the TBH antibody. This work was supported by US National Institutes of Health grants R01 MH0700000 to V.B., MH09883 to S.W., MH081982 to S.W. and GM084491 to M.J.A. M.J.A. was also supported by the Bill \& Melinda Gates Foundation.

\section{References}

1. Murchison CF, et al. A distinct role for norepinephrine in memory retrieval. Cell. 2004; 117:131143. [PubMed: 15066288]

2. $\mathrm{Hu} \mathrm{H}$, et al. Emotion enhances learning via norepinephrine regulation of AMPA-receptor trafficking. Cell. 2007; 131:160-173. [PubMed: 17923095]

3. Kuzmiski JB, Pittman QJ, Bains JS. Metaplasticity of hypothalamic synapses following in vivo challenge. Neuron. 2009; 62:839-849. [PubMed: 19555652]

4. Balfanz S, Strunker T, Frings S, Baumann A. A family of octopamine [corrected] receptors that specifically induce cyclic AMP production or $\mathrm{Ca}^{2+}$ release in Drosophila melanogaster. $\mathrm{J}$ Neurochem. 2005; 93:440-451. [PubMed: 15816867]

5. Hammer M, Menzel R. Multiple sites of associative odor learning as revealed by local brain microinjections of octopamine in honeybees. Learn Mem. 1998; 5:146-156. [PubMed: 10454379]

6. Schroll C, et al. Light-induced activation of distinct modulatory neurons triggers appetitive or aversive learning in Drosophila larvae. Curr Biol. 2006; 16:1741-1747. [PubMed: 16950113]

7. Schwaerzel M, et al. Dopamine and octopamine differentiate between aversive and appetitive olfactory memories in Drosophila. J Neurosci. 2003; 23:10495-10502. [PubMed: 14627633]

8. Zhou C, Rao Y. A subset of octopaminergic neurons are important for Drosophila aggression. Nat Neurosci. 2008; 11:1059-1067. [PubMed: 19160504]

9. Monastirioti M, Linn CE Jr, White K. Characterization of Drosophila tyramine beta-hydroxylase gene and isolation of mutant flies lacking octopamine. J Neurosci. 1996; 16:3900-3911. [PubMed: 8656284]

10. Suo S, Kimura Y, Van Tol HH. Starvation induces cAMP response element-binding proteindependent gene expression through octopamine-Gq signaling in Caenorhabditis elegans. J Neurosci. 2006; 26:10082-10090. [PubMed: 17021164]

11. Crocker A, Shahidullah M, Levitan IB, Sehgal A. Identification of a neural circuit that underlies the effects of octopamine on sleep:wake behavior. Neuron. 2010; 65:670-681. [PubMed: 20223202]

12. Breen CA, Atwood HL. Octopamine - a neurohormone with presynaptic activity-dependent effects at crayfish neuromuscular junctions. Nature. 1983; 303:716-718. [PubMed: 6406911]

13. Monastirioti M, et al. Octopamine immunoreactivity in the fruit fly Drosophila melanogaster. J Comp Neurol. 1995; 356:275-287. [PubMed: 7629319]

14. Schuster CM, Davis GW, Fetter RO, Goodman CS. Genetic dissection of structural and functional components of synaptic plasticity. II. Fasciclin II controls presynaptic structural plasticity. Neuron. 1996; 17:655-667. [PubMed: 8893023]

15. Marqués G, Zhang B. Retrograde signaling that regulates synaptic development and function at the Drosophila neuromuscular junction. Int Rev Neurobiol. 2006; 75:267-285. [PubMed: 17137932]

16. Korkut C, Budnik V. WNTs tune up the neuromuscular junction. Nat Rev Neurosci. 2009; 10:627634. [PubMed: 19693027]

17. Steinert JR, et al. Experience-dependent formation and recruitment of large vesicles from reserve pool. Neuron. 2006; 50:723-733. [PubMed: 16731511]

18. Sigrist SJ, et al. Experience-dependent strengthening of Drosophila neuromuscular junctions. J Neurosci. 2003; 23:6546-6556. [PubMed: 12878696] 
19. Budnik V, Zhong Y, Wu CF. Morphological plasticity of motor axons in Drosophila mutants with altered excitability. J Neurosci. 1990; 10:3754-3768. [PubMed: 1700086]

20. Ataman B, et al. Rapid activity-dependent modifications in synaptic structure and function require bidirectional wnt signaling. Neuron. 2008; 57:705-718. [PubMed: 18341991]

21. Saraswati S, Fox LE, Soil DR, Wu CF. Tyramine and octopamine have opposite effects on the locomotion of Drosophila larvae. J Neurobiol. 2004; 58:425-441. [PubMed: 14978721]

22. Davenport AP, Evans PD. Changes in haemolymph octopamine levels associated with food deprivation in the locust, Schistocerca gregaria. Physiol Entomol. 1984; 9:269-274.

23. Hardie SL, Zhang JX, Hirsh J. Trace amines differentially regulate adult locomotor activity, cocaine sensitivity, and female fertility in Drosophila melanogaster. Dev Neurobiol. 2007; 67:1396-1405. [PubMed: 17638385]

24. Kittel RJ, et al. Bruchpilot promotes active zone assembly, $\mathrm{Ca}^{2+}$ channel clustering, and vesicle release. Science. 2006; 312:1051-1054. [PubMed: 16614170]

25. Rasse TM, et al. Glutamate receptor dynamics organizing synapse formation In vivo. Nat Neurosci. 2005; 8:898-905. [PubMed: 16136672]

26. Fouquet W, et al. Maturation of active zone assembly by Drosophila Bruchpilot. J Cell Biol. 2009; 186:129-145. [PubMed: 19596851]

27. Mosca TJ, Carrillo RA, White BH, Keshishian H. Dissection of synaptic excitability phenotypes by using a dominant-negative Shaker K* channel subunit. Proc Natl Acad Sci USA. 2005; 102:3477-3482. [PubMed: 15728380]

28. Kitamoto T. Conditional modification of behavior in Drosophila by targeted expression of a temperature-sensitive shibire allele in defined neurons. J Neurobiol. 2001; 47:81-92. [PubMed: 11291099]

29. White BH, et al. Targeted attenuation of electrical activity in Drosophila using a genetically modified K(+) channel. Neuron. 2001; 31:699-711. [PubMed: 11567611]

30. Paradis S, Sweeney ST, Davis GW. Homeostatic control of presynaptic release is triggered by postsynaptic membrane depolarization. Neuron. 2001; 30:737-749. [PubMed: 11430807]

31. McGuire SE, Mao Z, Davis RL. Spatiotemporal gene expression targeting with the TARGET and gene-switch systems in Drosophila. Sci STKE. 2004; 2004:pl6. [PubMed: 14970377]

32. Han KA, Millar NS, Davis RL. A novel octopamine receptor with preferential expression in Drosophila mushroom bodies. J Neurosci. 1998; 18:3650-3658. [PubMed: 9570796]

33. Zhong Y, Budnik V, Wu CF. Synaptic plasticity in Drosophila memory and hyperexcitable mutants: role of cAMP cascade. J Neurosci. 1992; 12:644-651. [PubMed: 1371316]

34. Schröder-Lang S, et al. Fast manipulation of cellular cAMP level by light in vivo. Nat Methods. 2007; 4:39-42. [PubMed: 17128267]

35. Benito E, Barco A. CREB's control of intrinsic and synaptic plasticity: implications for CREBdependent memory models. Trends Neurosci. 2010; 33:230-240. [PubMed: 20223527]

36. Perazzona B, Isabel G, Preat T, Davis RL. The role of cAMP response element-binding protein in Drosophila long-term memory. J Neurosci. 2004; 24:8823-8828. [PubMed: 15470148]

37. Lee HG, et al. Octopamine receptor OAMB is required for ovulation in Drosophila melanogaster. Dev Biol. 2003; 264:179-190. [PubMed: 14623240]

38. Schuster CM, Davis GW, Fetter RD, Goodman CS. Genetic dissection of structural and functional components of synaptic plasticity. I. Fasciclin II controls synaptic stabilization and growth. Neuron. 1996; 17:641-654. [PubMed: 8893022]

39. O’Dell TJ, Connor SA, Gelinas JN, Nguyen PV. Viagra for your synapses: enhancement of hippocampal long-term potentiation by activation of beta-adrenergic receptors. Cell Signal. 2010; 22:728-736. [PubMed: 20043991]

40. Roeder T. Tyramine and octopamine: ruling behavior and metabolism. Annu Rev Entomol. 2005; 50:447-477. [PubMed: 15355245]

41. Howell KM, Evans PD. The characterization of presynaptic octopamine receptors modulating octopamine release from an identified neurone in the locust. J Exp Biol. 1998; 201:2053-2060. [PubMed: 9622577] 
42. Floresco SB, et al. Afferent modulation of dopamine neuron firing differentially regulates tonic and phasic dopamine transmission. Nat Neurosci. 2003; 6:968-973. [PubMed: 12897785]

43. Aamodt SM, Constantine-Paton M. The role of neural activity in synaptic development and its implications for adult brain function. Adv Neurol. 1999; 79:133-144. [PubMed: 10514810]

44. Landgraf M, Bossing T, Technau GM, Bate M. The origin, location, and projections of the embryonic abdominal motorneurons of Drosophila. J Neurosci. 1997; 17:9642-9655. [PubMed: 9391019]

45. Takizawa E, Komatsu A, Tsujimura H. Identification of common excitatory motoneurons in Drosophila melanogaster larvae. Zoolog Sci. 2007; 24:504-513. [PubMed: 17867850]

46. Nagaya Y, Kutsukake M, Chigusa SI, Komatsu A. A trace amine, tyramine, functions as a neuromodulator in Drosophila melanogaster. Neurosci Lett. 2002; 329:324-328. [PubMed: 12183041]

47. Kutsukake M, Komatsu A, Yamamoto D, Ishiwa-Chigusa S. A tyramine receptor gene mutation causes a defective olfactory behavior in Drosophila melanogaster. Gene. 2000; 245:31-42. [PubMed: 10713442]

48. Nishikawa K, Kidokoro Y. Octopamine inhibits synaptic transmission at the larval neuromuscular junction in Drosophila melanogaster. Brain Res. 1999; 837:67-74. [PubMed: 10433989]

49. McNabb SL, et al. Disruption of a behavioral sequence by targeted death of peptidergic neurons in Drosophila. Neuron. 1997; 19:813-823. [PubMed: 9354328]

50. Cheung US, Shayan AJ, Boulianne GL, Atwood HL. Drosophila larval neuromuscular junction's responses to reduction of cAMP in the nervous system. J Neurobiol. 1999; 40:1-13. [PubMed: 10398067] 
a

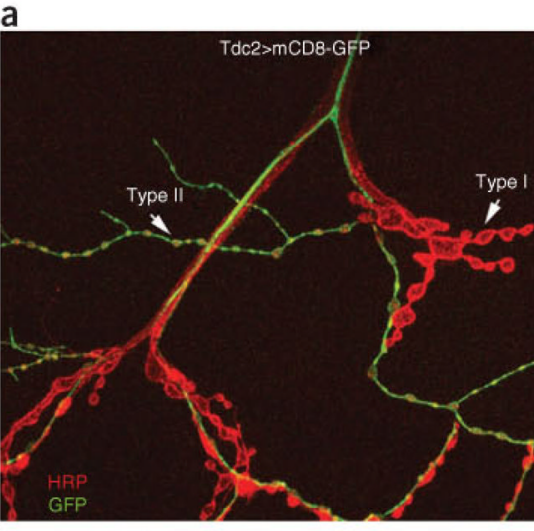

C ${ }_{8} \sqrt{* * *}$ d b
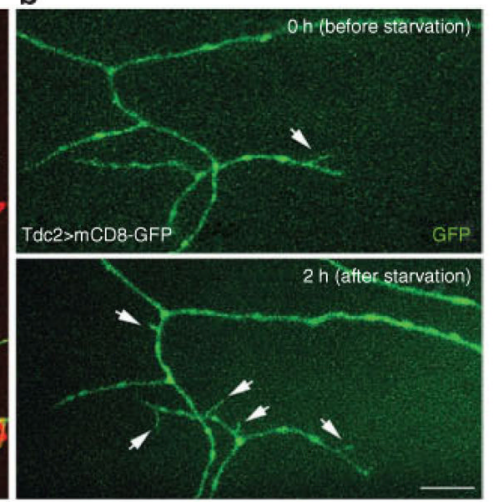

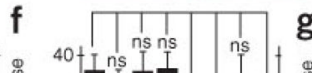

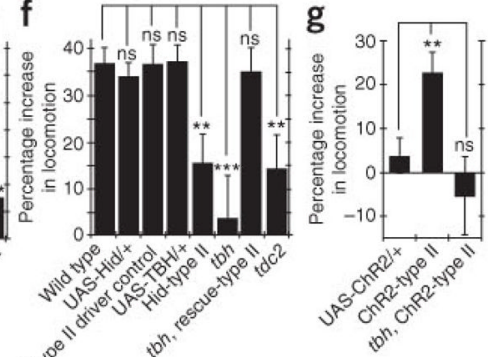

i
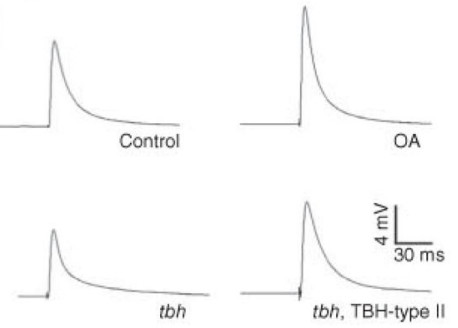

Figure 1.

Food-deprivation increase in larval locomotion is correlated with synaptopod formation at type II arbors, (a) NMJs at muscles 12 and 13 of a third-instar larva expressing mCD8-GFP in type II motor neurons, showing type I and type II endings (arrows), labeled with anti-HRP and anti-GFP. (b) Live imaging of type II endings through the cuticle of intact larvae before and after 2-h starvation. Arrows, synaptopods. (c) Locomotor speed of wild-type (Canton-S) larvae before and after 2-h starvation $(n=31,26)$. (d) Number of synaptopods (pods) in fed and 2-h starved intact Tdc2>mCD8-GFP larvae $(n=10,10)$. (e) Locomotor speed in the indicated genotypes $(n=31,23,29,15,34,18,25)$. (f) Percentage increase in locomotor speed in response to starvation in the indicated genotypes $(n=26,25,15,14,38,14,25)$. (g) Percentage increase in locomotor speed in response to light stimulation in the indicated genotypes $(n=20,20,16)$. (h) Ratio of EJP and mEJP amplitude upon bath application of $10 \mu \mathrm{M}$ octopamine ( $n=10$ animals). (i) EJP and mEJP amplitude in $t b h$ mutants ( $n=6,5$ and 5 animals, respectively). (j) Representative EJP traces in the indicated conditions. Scale bar, $8.5 \mu \mathrm{m}(\mathbf{a}), 7.5 \mu \mathrm{m}(\mathbf{b})$. Error bars represent s.e.m. ${ }^{* * *} P \leq 0.0001,{ }^{* *} P \leq 0.001,{ }^{*} P<$ 0.05 . 

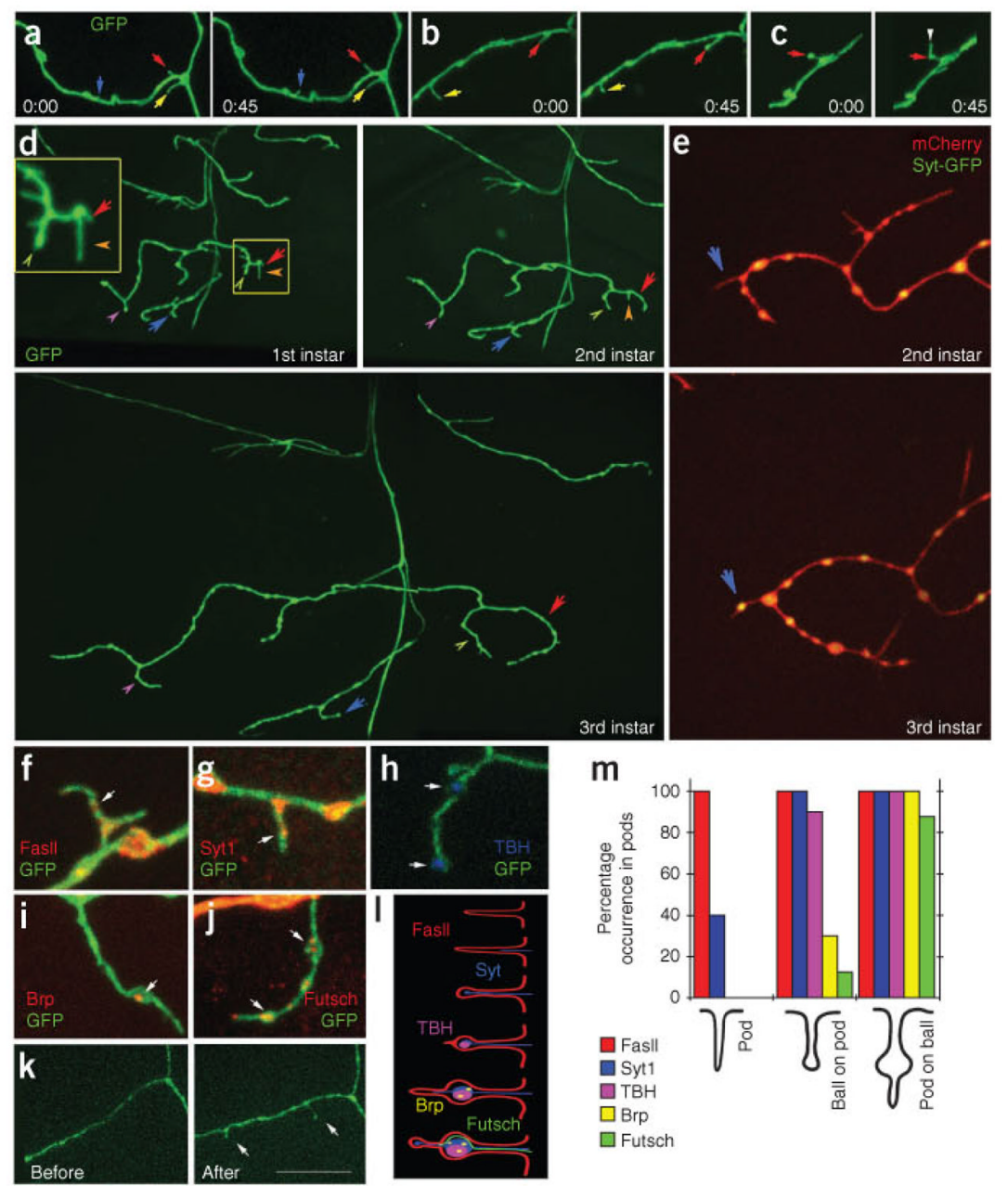

Figure 2.

Stepwise development of synaptopods. (a-c) Time lapse imaging of synaptopods in Tdc2>mCD8-GFP larvae showing the extension of synaptopods (a. arrows), the formation of varicosities at the tip of synaptopods (b, arrows) and the formation of a secondary synaptopod (c, arrowhead) from a newly formed varicosity (arrow). Images of the same NMJs were taken $45 \mathrm{~s}$ apart, (d) Developmental time-lapse imaging of the same NMJ through the cuticle of first, second and third-instar larvae. Red arrow, a synaptopod developed into an entire branch. Orange arrow, a synaptopod was eliminated at third instar. Blue arrow, a synaptopod developed into a varicosity. Yellow and purple arrows, a varicosity developed into a new branch. (e) Time-lapse imaging as in $\mathbf{d}$ in animals expressing mCherry and Syt1-GFP in type II endings in second and third instar. Arrow, synaptopod that acquired Syt1-containing varicosity, $(\mathbf{f}-\mathbf{j})$ Sequence of protein addition to an extending type II branch in Tdc2>mCD8- GFP preparations triple labeled with antibodies to GFP, TBH and FasII (f), Syt1 (g), TBH (h), Brp (i) or Futsch (j). Arrows, sites of protein localization at synaptopods or newly formed varicosities. (k) Live imaging of synaptopods through the cuticle of intact Tdc2>mCD8-GFP larvae before and after starvation, showing the formation of varicosities (arrows) on top of synaptopods after starvation. (l) Sequence of synaptic protein addition at developing type II endings. (m) Percentage of synaptopods containing the indicated proteins at the stages of synaptopod, varicosity at the tip of a synaptopod (ball on pod) and secondary synaptopod (pod on ball). $n$ (number of pod 
structures) is 30 for FasII, 30 for Syt1, 20 for TBH, 20 for Brp and 10 for Futsch. Scale bars, $10 \mu \mathrm{m}(\mathbf{a}-\mathbf{c}), 22 \mu \mathrm{m}(\mathbf{d}), 8.5 \mu \mathrm{m}(\mathbf{e}), 2.5 \mu \mathrm{m}(\mathbf{f}-\mathbf{j})$ and $12 \mu \mathrm{m}(\mathbf{k})$. 

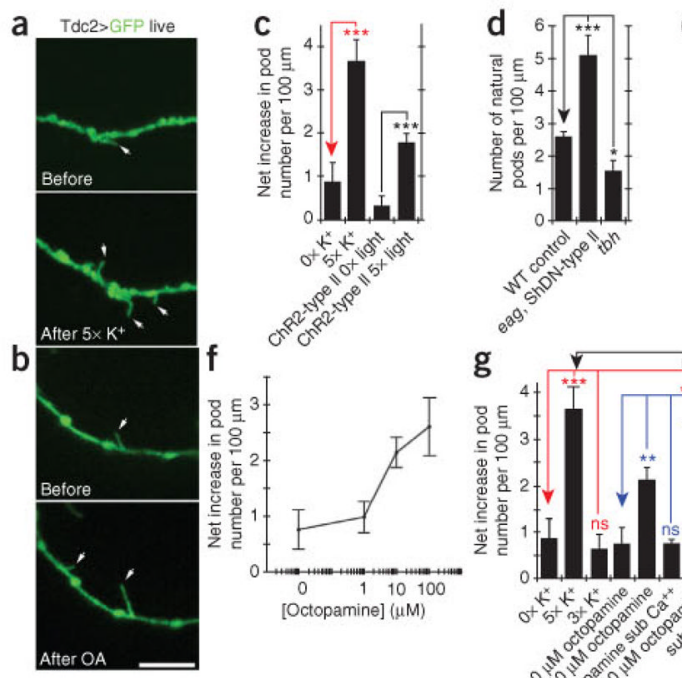

e
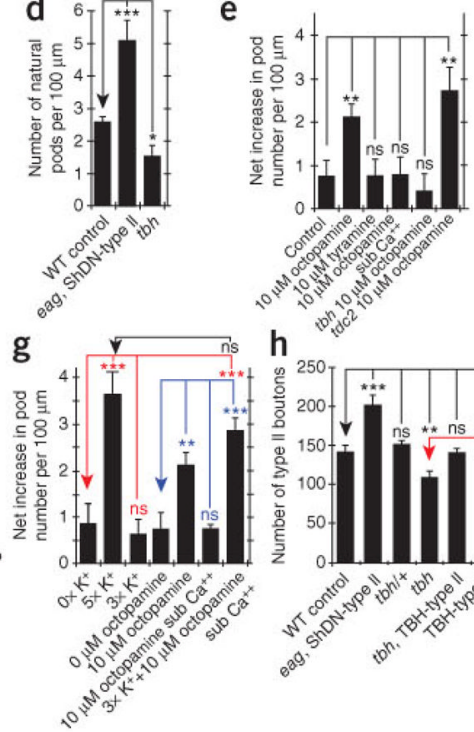

h

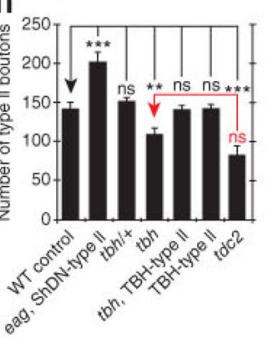

Figure 3.

Electrical activity and octopamine regulate the extension of synaptopods. (a,b) Live imaging of synaptopods before and after stimulation with high $\mathrm{K}^{+}(\mathbf{a})$ or octopamine $(\mathbf{b})$ in Tdc2 $>$ mCD8-GFP larvae. (c) Net increase in synaptopod number $\sim 2 \mathrm{~h}$ after $\mathrm{K}^{+}$or ChR2 stimulation (see Online Methods for details; $n=8,10,10,11$ ). (d) Number of natural synaptopods in Tdc2>mCD8-GFP (WT control) and indicated genotypes $(n=31,11,31)$. (e) Net increase in synaptopod number in Tdc2>mCD8-GFP control (no drug) and preparations exposed to the indicated drugs $(n=11,13,8,18)$. (f) Net increase in the number of synaptopods in response to different concentrations of octopamine in Tdc2>GFP larvae $(\mathrm{n}=11,7,9,11)$. (g) Net increase in synaptopod number at subthreshold concentration of octopamine or $\mathrm{K}^{+}$depolarization in the presence of $0.1 \mathrm{mM} \mathrm{Ca}^{2+}\left(\mathrm{sub}-\mathrm{Ca}^{2+}\right)$ in Tdc2>mCD8-GFP larvae $(\mathrm{n}=8,10,11,11.13,18,13)$. (h) Number of type II boutons at muscle 12 (A3) of third-instar larvae in the indicated genotypes $(n=16,10,15,14,12,6)$; wild-type, Canton-S. (i) Percentage increase in locomotor speed in response to food deprivation in the indicated genotypes $(n=31,20,34,18,25)$; WT control, Canton-S. Scale bar, $7 \mu \mathrm{m}(\mathbf{a}, \mathbf{b})$. Error bars represent s.e.m. ${ }^{* * *} P \leq 0.0001,{ }^{* *} P \leq 0.001,{ }^{*} P<0.05$. 


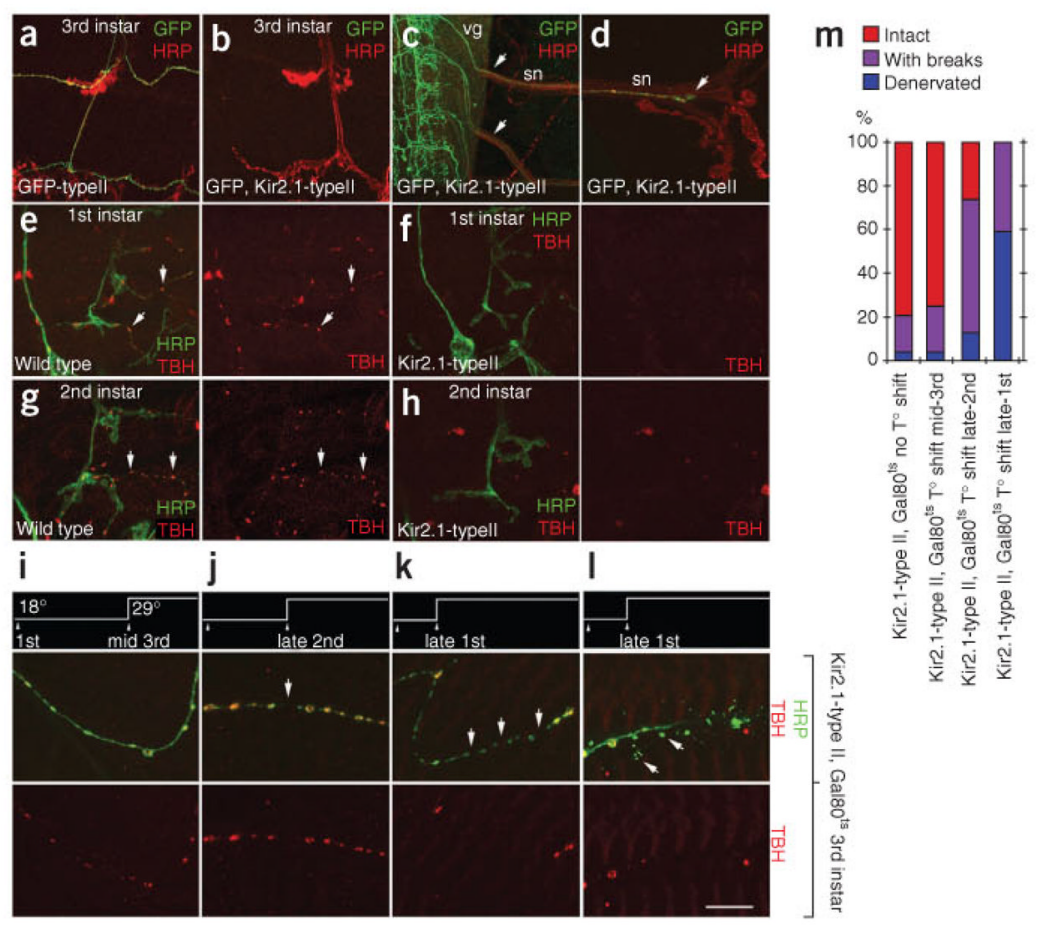

Figure 4.

Innervation and maintenance of type II arbors depends on activity. (a,b) NMJs at muscles 12 and 13 in preparations expressing mCD8-GFP in octopamine neurons and double labeled with anti-GFP and anti-HRP antibodies in Tdc2>mCD8-GFP (a) and Tdc2>mCD8-GFP, Kir2.1 (b) third-instar larvae, (c,d) Type II motor neuron axons labeled with anti-GFP and anti-HRP emerging from the CNS at the segmental nerves (c, arrows) and terminating close to the NMJ (d, arrow) in GFP, Kir2.1-type II preparations stained with antibodies to HRP and GFP. (e-h) NMJs at muscles 12 and 13 in preparations expressing mCD8-GFP in octopamine neurons and double labeled with anti-HRP and anti-TBH antibodies in wild-type $(\mathbf{e , g})$ and Tdc2>Kir2.1 (f,h) larvae at the first (e,f) and second-instar (g,h) larval stages. Arrows point to type II boutons. (i-1) Third-instar type II NMJs from Kir2.1-type II, Gal80 ${ }^{\text {ts }}$ larvae shifted to $29^{\circ} \mathrm{C}$ at the stages indicated in the time scale at the top of each panel, double stained with anti-HRP and anti-TBH. Arrows point to breaks in the arbors or debris. (m) Percentage of intact, broken or absent (denervated) type II NMJs in Tdc2>Kir2.1, TubPGal80 $0^{\text {ts }}$ (Kir2.1-type II, Gal80 ${ }^{\text {ts }}$ ) larvae shifted to $29^{\circ} \mathrm{C}$ at the indicated stages $(n=25,24$, 46, 22). Scale bar, $24 \mu \mathrm{m}$ (a,b), $12 \mu \mathrm{m}(\mathbf{d}, \mathbf{e}-\mathbf{h}), 38 \mu \mathrm{m}$ (c) and $10 \mu \mathrm{m}$ (i-l). 

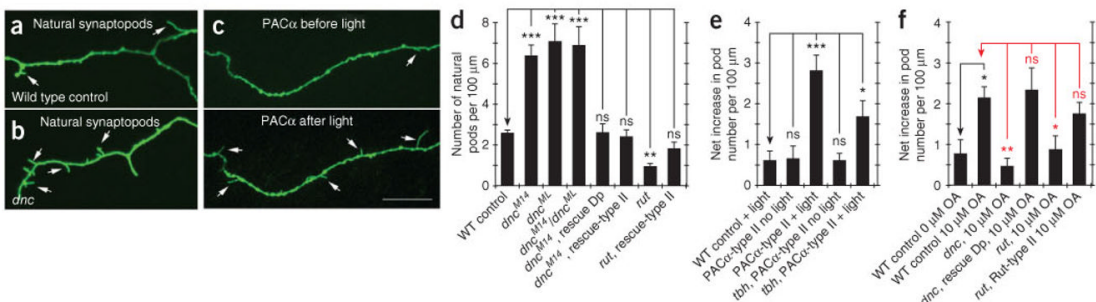

g

h
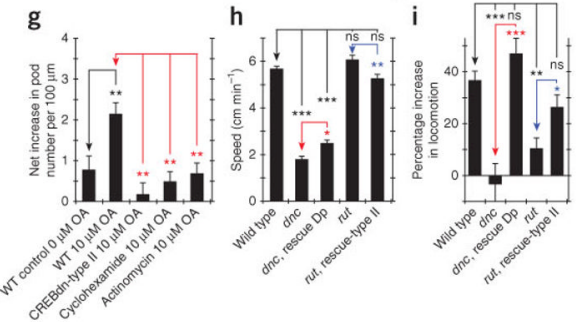

Figure 5.

Synaptopod extension is regulated by the cAMP pathway and requires new protein synthesis and CREB. (a-c) Live imaging of synaptopods (arrows) in Tdc $2>\mathrm{mCD} 8-\mathrm{GFP}$ (a, WT control), $d n c^{M 14}$, Tdc2>mCD8-GFP flies (b) and Tdc2>mCD8- GFP, PACa flies (c) before and after light stimulation. (d) Number of natural synaptopods in the indicated genotypes $(n=175,21,11,8,17,11,24,11)$. (e-g) Net increase in synaptopod number in preparations expressing PACa in wild type and $t b h$ mutant background and subjected to the indicated light procedures $(\mathbf{e}, n=10,12,12,11,12)$, upon application of octopamine (OA) in the indicated genotypes $(\mathbf{f}, n=11,13,10.11,12,8)$ and in Tdc2>mCD8-GFP preparations treated with actinomycin or cycloheximide, or expressing Tdc2>CREBdn and exposed to octopamine as indicated (g, $n=11,13,18,11,13$; see Online Methods for details of PAC and octopamine stimulation procedures). (h) Locomotor speed of the indicated genotypes ( $n$ $=31,18,15,21,15)$. (i) Percentage increase in locomotor speed in response to food deprivation in the indicated genotypes $(n=26,18,15,21,15)$. Scale bar, $14 \mu \mathrm{m}(\mathbf{a}-\mathbf{c})$. Error bars represent s.e.m. ${ }^{* * *} P \leq 0.0001,{ }^{* *} P \leq 0.001,{ }^{*} P<0.05$. 

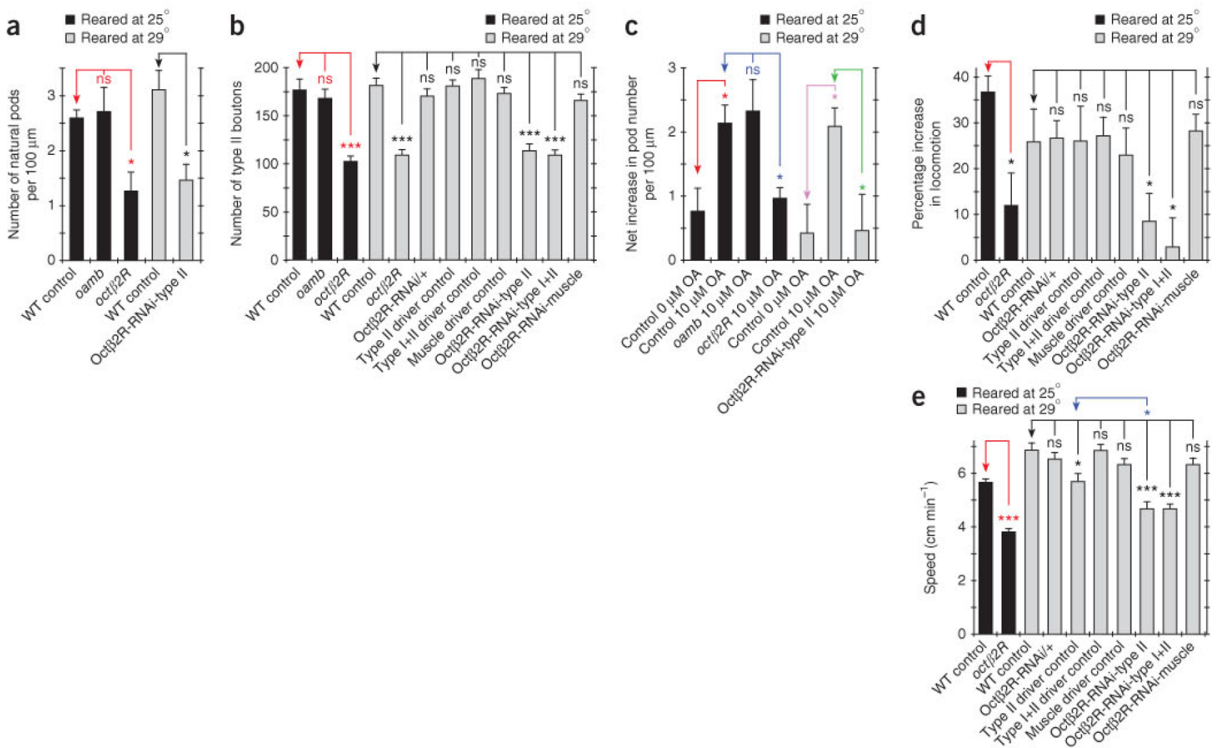

Figure 6.

Presynaptic Oct $\beta 2 R$ autoreceptors, but not OAMB receptors, regulate the growth of type II arbors. (a) Number of natural synaptopods in the indicated genotypes $(n=175,12,13,25$, 14); WT control is Tdc2>mCD8-GFP. (b) Number of type II boutons in the indicated genotypes $(n=16,12,16,16,15,11,11,10,12,12,13,12)$; WT control is Canton-S; type

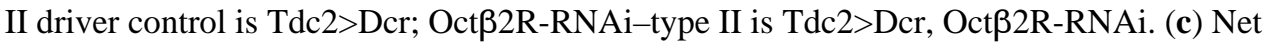
increase in the number of synaptopods in the indicated conditions and genotypes $(n=11,13$, 11, 10, 12, 11, 14; WT control isTdc2>mCD8-GFP). (d) Percentage increase in locomotor speed in the indicated genotypes in response to food deprivation $(n=26,16,13,15,13,17$, $15,13,13,15)$. (e) Locomotor speed in the indicated genotypes $(n=31,20,17,17,15,18$, $17,13,13,17)$. For $\mathbf{d}$ and $\mathbf{e}$, WT control is Canton-S; type II driver control is Tdc2>Dcr; type I+II driver control is C380>Dcr; muscle driver control is C57>Dcr; Oct $\beta 2 \mathrm{R}-\mathrm{RNAi}-$ type II is Tdc2>Dcr, Oct $\beta 2 R-R N A i$; Oct $\beta 2 R-R N A i-t y p e ~ I+I I$ is C380>Dcr, Oct $\beta 2 R-R N A i$; Oct $\beta 2 R-R N A i-m u s c l e ~ i s ~ C 57>D c r$, Oct $\beta 2 R-R N A i$. Error bars represent s.e.m. ${ }^{* * *} P \leq$ $0.0001,{ }^{*} P<0.05$. 

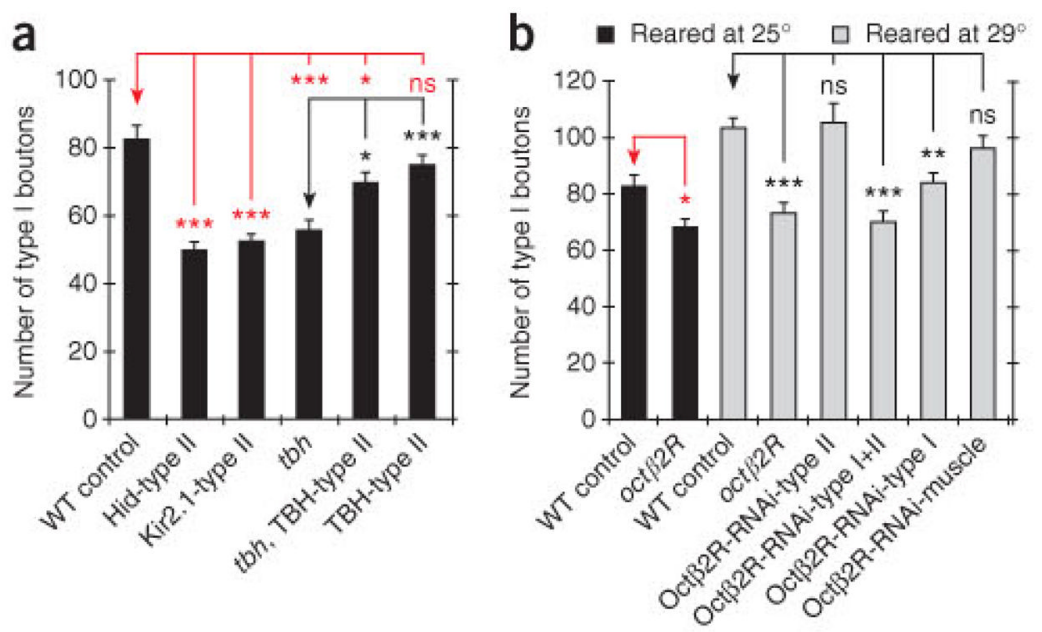

Figure 7.

Type II motor neurons regulate the growth of type I arbors. (a) Number of type I boutons at muscle 6 and 7 (A3) in third-instar larvae after eliminating either type II motor neurons or octopamine production from type II motor neurons $(n=12,17,21,18,20,13)$. (b) Same data from larvae with decreased oct $\beta 2 \mathrm{R}$ levels $(N=12,36,17,18,12,13,11)$. WT controls are Canton-S. Error bars represent s.e.m. ${ }^{* * *} P \leq 0.0001,{ }^{* *} P \leq 0.001,{ }^{*} P<0.05$. 